\title{
How to be a great dad: Parental care in a flock of greater flamingo (Phoenicopterus roseus)
}

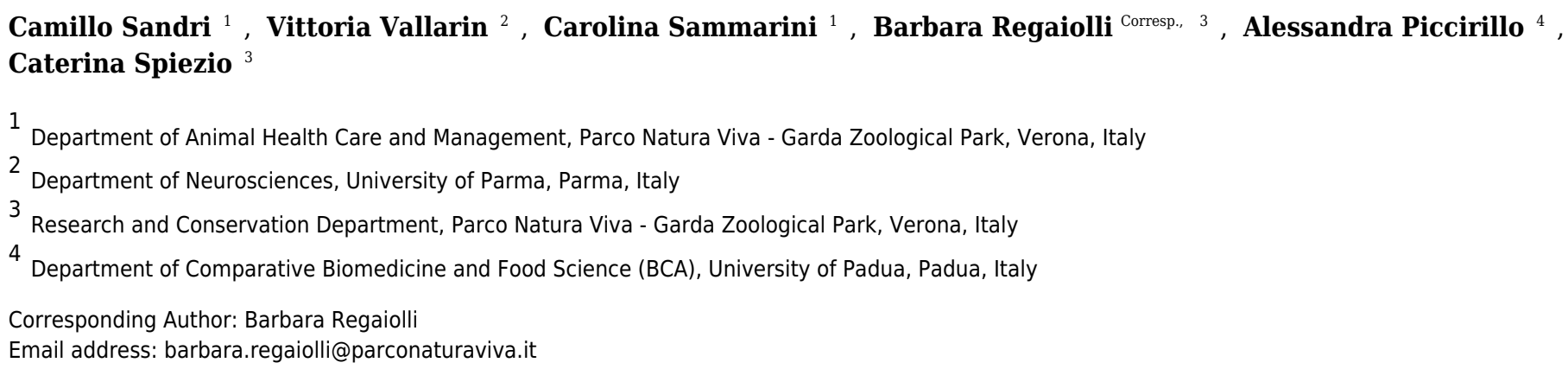

In the last years, studies on captive greater flamingos have increased. Research on captive animals is important to improve the knowledge on these species and to improve their exsitu and in-situ conservation. The aim of the present study was to investigate the parental behaviour of a captive colony of greater flamingo hosted at Parco Natura Viva, an Italian zoological garden to improve the knowledge on this species in zoos. In particular, the present study investigated and compared the parental care of females and males in 35 breeding pairs of greater flamingos. For each pair, we collected durations of parental care behaviour of both females and males, recording their position in relation to the nest (near the nest, on the nest, away from the nest) and individual and social behaviours performed. First, both partners were involved in parental care and displayed species-specific behaviours reported in the wild. The main results were that males spent more time than females on the nest $(P=0.010)$ and near it $(P=0.0001)$ and were more aggressive toward other flamingos than females, both when sitting on the nest $(P=0.003)$ and when near the nest $(P=0.0003)$. Therefore, male flamingos seem to be more involved in incubation duties and nest protection than females. This kind of research is important not only to expand the knowledge on bird species such as flamingos, but also to improve their husbandry and breeding in controlled environment. Indeed, understanding animal behaviour allows us to gain insights into their individual and social needs, addressing potential animal welfare issues. 
1 RUNNING HEAD: PARENTAL CARE IN GREATER FLAMINGO

2

3 How to be a great dad: Parental care in a flock of greater flamingo (Phoenicopterus roseus)

$4 \quad$ Camillo Sandri ${ }^{1}$; Vittoria Vallarin ${ }^{2}$; Carolina Sammarini ${ }^{1}$; Barbara Regaiolli ${ }^{*}$; Alessandra

Piccirillo $^{4}$; Caterina Spiezio ${ }^{3}$ 


\section{Abstract}

In the last years, studies on captive greater flamingos have increased. Research on captive animals is important to improve the knowledge on these species and to improve their ex-situ and in-situ conservation. The aim of the present study was to investigate the parental behaviour of a captive colony of greater flamingo hosted at Parco Natura Viva, an Italian zoological garden to improve the knowledge on this species in zoos. In particular, the present study investigated and compared the parental care of females and males in 35 breeding pairs of greater flamingos. For each pair, we collected durations of parental care behaviour of both females and males, recording their position in relation to the nest (near the nest, on the nest, away from the nest) and individual and social behaviours performed. First, both partners were involved in parental care and displayed species-specific behaviours reported in the wild. The main results were that males spent more time than females on the nest $(P=0.010)$ and near it $(P=0.0001)$ and were more aggressive toward other flamingos than females, both when sitting on the nest $(P=0.003)$ and when near the nest $(P=0.0003)$. Therefore, male flamingos seem to be more involved in incubation duties and nest protection than females. This kind of research is important not only to expand the knowledge on bird species such as flamingos, but also to improve their husbandry and breeding in controlled environment. Indeed, understanding animal behaviour allows us to gain insights into their individual and social needs, addressing potential animal welfare issues.

40 Word count: 2701 


\section{INTRODUCTION}

42 Studies on wild flamingos are challenging to carry out. Indeed, flamingos are highly gregarious birds that live and breed in large dense flocks often including thousands of pairs (Pickering et al., 1992; Johnson \& Cèzlly, 2009). Obtaining information and data on their behaviour in the wild is therefore difficult due to constraints such as individual identification and approach to the birds (Studer-Thiersch, 1975; 2000; King, 2000). Zoo environments provide researchers with relative accessibility to the animals and important to obtain data on exotic animals, providing useful insights into the biology of their wild counterparts (Hosey. Melfi \& Pankhurst, 2013). Despite research in zoological gardens might be compromised by confounding variables such as reduced sample size and human presence, the zoo environment houses animals in naturalistic group and enclosures, allowing the collection of data having a great biological relevance (Hosey et al., 2013). For these reasons, together with long-term studies on wild flamingo flocks, research on zoo colonies might be valuable and complementary to improve the knowledge on the ethology, morphology, physiology and endocrinology of these species (King, 2000). Moreover, studying the behaviour of flamingos in both wild and captive settings is important not only to improve our knowledge on the species biology but also for the implementation of their husbandry and their breeding (King, 2000; Melfi, 2009; Rose et al., 2014). Indeed, understanding species-specific behaviours, their function and causes, would be useful to know the animal feelings, preferences and needs, detecting and addressing potential welfare issues (Mench, 1998; Hosey et al., 2013). Greater flamingos display sexual body size dimorphism as males are larger than females

61 (Johnson \& Cèzilly, 2009). Greater flamingos are serially monogamous birds but they can form

62 long-term pair bonds, at least in captivity (Pickering, 1992; Bagemihl, 1999; Johnson \& Cèzilly, 63 2009; Rose \& Croft, 2015). Both partners work together to build a nest, in which the female lays 
64 a single egg. The nest is usually a mound made of a mixture of mud/soil and sand, with a

65

66

67

68

69

70

71

72

concave centre. It is generally built on an island or on the coastline of a lake (Studer-Thiersch, 1975; Beletsky, 2006; Cezilly, 1993; Elphick, 2014). Before egg laying, the male is primarily involved in nest building, but the female takes over as the laying time approaches. The nest building activity of both partners proceeds also during the first two weeks of incubation, leading to an increase in the nest height (Studer-Thiersch, 1975).

After copulatory behaviour, the female lays one egg in the nest. Then, both females and males take part in the incubation, lasting from 27 to 31 days (Beletsky, 2006; Cèzilly, 1993; Elphick, 2014). Male greater flamingos have been found to make a greater effort in incubation (Rendòn-Martos et al., 2000). When incubating the egg, flamingos display different behaviours, such as standing, stretching the wings, preening, self-scratching and looking at the nest (StuderThiersch, 1975). In addition, they take care of the egg, moving it with the bill. Flamingos could either stand or sit on the nest (Studer-Thiersch, 1975). While nesting, individuals of a pair including both the incubating bird and the non-incubating bird may perform aggressive display behaviour (Studer-Thiersch, 1975).

The aim of the present study was to investigate the parental behaviour of a captive colony of greater flamingo hosted at Parco Natura Viva, an Italian zoological garden to improve the knowledge on this species in zoos. In particular, the present study investigated and compared the parental care behaviour of females and males in a flock of greater flamingos, to verify the presence of both qualitative and quantitative differences between the study birds and their wild counterparts. The results of the study are discussed with the behavioural pattern shown by greater flamingos in the wild, to suggest strategies to improve the husbandry of this species in captivity and safeguard its welfare. 


\section{MATERIALS AND METHODS}

\section{Study subjects and area}

The study was carried out in a flock of 147 greater flamingos of different ages (from 1 to over 20 years), 70 females and 77 males, housed at Parco Natura Viva - Garda Zoological Park in Italy, in a 1,100 $\mathrm{m}^{2}$ open-topped enclosure. The study subjects were 35 heterosexual breeding pairs (approximately 50\% of the study flock), during the peak of their breeding activity. The colony was composed of several juvenile immature individuals that were not involved in pair formation. Same-sex pairs and trios incubating an egg were not involved in the current study. The enclosure was composed of a muddy area and a grassy area. The muddy area surrounded a water pool with two islands, used by flamingos to build their nest mounds and rear the chicks. The basal structure of the nest mound was built by humans, whereas flamingo pairs completed the nest construction properly, using mud, soil and sand naturally present in the enclosure. The nests had a density of approximately 2.5 nests $/ \mathrm{m}^{2}$. In particular, the nesting island had an area with an higher density of nests while others had a lower density. Trees, bushes and rocks were present in the enclosure, together with a wooden house to provide the flamingos with protection from weather conditions and a long feeding station. To minimize human disturbance, food was administered to the flamingos once a day in the feeding point. No interactions between humans and flamingos were allowed. The flamingo diet was composed of a specific pellet, containing cereals, vegetables, oils and fats, algae, shellfish, vitamins and mineral salts.

Flamingos were identified through a ring on one leg. The ring differed in colour and letters (three-letter combination). At the time of the study, the density of the flamingos in the enclosure was 0.13 individuals $/ \mathrm{m}^{2}$. Subjects of the study were pairs that incubated an egg in the 2016 breeding season $(\mathrm{N}=35)$. To avoid excessive inbreeding, juvenile individuals were 
110 transferred regularly (approximately every five to six years) to new flocks in other zoological

111 institutions. However, no bird transfers from and to other zoological gardens were done during

112 the study period and in adjacent breeding seasons and the flock increased only due to flamingo

113 reproductive activity. The study flock size ranged from 88 greater flamingos in 2012 to 147 in

114 2016, the year of the current study (Sandri et al., submitted).

115 The study was carried out using non-invasive techniques, through live observation of the

116 birds. The research procedure was in accordance with the EU Directive 2010/63/EU for animal

117 research.

118 Procedure and data collection

119 Subjects of the study were heterosexual breeding pairs and data collection started at the moment

120 when a female laid an egg. For each pair, a total of twenty 10-minute sessions were carried out

121 during the incubation period. In particular, for each pair two sessions per day were done, one in

122 the morning (between 9.00 and 12.00) and one in the afternoon (between 14.00 and 17.00). To

123 have data distributed over the entire study period, the observation sessions were carried out

124 randomly within the incubation period. Data were collected using a continuous focal animal

125 sampling method to record durations of behaviours performed by each flamingo partner

126 (Altmann, 1974). One observer (V.V.) collected the data on all the study breeding pairs. Before

127 commencing systematically data collection, the observer spent one month observing the

128 flamingo behaviour whilst the birds get used to her. In addition, she carried out preliminary

129 observations $(40 \mathrm{~h})$ in order to establish the data collection methods and to prepare an ethogram

130 to be used to collect behavioural data. The ethogram (Tab. 1) was made basing on the studies of

131 Studer-Thiersch (1975), Schmitz \& Baldassarre (1992), Brown \& King (2005), Johnson \& 
132 Cèzilly (2009), Hauber (2014) and was adjusted to this study basing on the preliminary 133 observations.

Data collection was carried out following Altmann (1974), using the focal sub-group

135 sampling method in the presence of an egg on the nest. The chosen sub-group was the pair and 136 the behaviour of each partner of the pair was collected during the session. For each pair, we 137 conducted observations of parental care behaviour of both female and male, recording the 138 position of the bird in relation to the nest and the behavioural category performed. During each session continuous observation of each partner was done when flamingos were on the nest or near the nest. We collected durations of individual and social behaviours performed by male and female partners. In addition, to be accurate, when one partner was away from the nest and not under observation, the duration of "away from the nest" was also collected. In other words, we did not collect the behaviour of the subject away from the focal point as "under most circumstances, the only condition under which such a record can be obtained is that in which all the individuals in the sample group are continuously visible throughout the sample period" (Altmann, 1974). flamingo partner near the nest (less than $150 \mathrm{~cm}$, which is approximately the higher flamingo body length; del Hoyo et al., 1992), on the nest or away from the nest (>150 cm). observed in a variable order. The focal-sampling of each pair started at precise moment of the morning and of the afternoon, following a regular schedule. Over the data collection sessions, to 12.00 and from 14.00 to 17.00 . 
155 As the observed behavioural categories were mutually exclusive, not overlapping, during focal-

156 pair sampling we recorded the transition times, meaning that a behaviour ended when a new

157 behaviour started.

158 Statistical analysis

159 Kolmogorov-Smirnov goodness-of-fit tests revealed that not all data were normally

160 distributed. Therefore, non-parametric statistic tests were used. In particular, Mann-Whitney tests

161 were run to compare the duration of both positions and behaviours between females and males.

162 RESULTS

\section{Reproduction of the greater flamingo flock in 2016}

164 First, to be able to interpret more in deep the results on greater flamingo parental care behaviour we provide some data on the reproduction of the study flock in 2016 breeding season. In the current study, 27 out of 35 breeding pairs had a chick (77\%). Moreover, within the 27 successful pairs, only two of them $(7.4 \%)$ had to lay more than once before obtaining a successful hatch.

Position of female and male flamingos in relation to the nest in different positions relatively to the nest. Females spent on average $8.7 \%$ of the observed time near the nest, $44.7 \%$ on the nest and $46.6 \%$ away from the nest whereas males spent on average $25.7 \%$ of the observed time near the nest, $55.3 \%$ on the nest and $19 \%$ away from the nest (see

Fig. 1, 2 and 3 for medians and IQR). Mann-Whitney tests revealed that males were near the nest 174 (Fig. 1) and on the nest (Fig. 2) significantly more than females $(U=140, P<0.00001$, and $U=$ $175393, P=0.010, \mathrm{~N}_{I}=\mathrm{N}_{2}=35$, respectively). On the contrary, males were away from the nest 176 significantly less than females (Fig. 3) $\left(U=121.5, P<0.00001, \mathrm{~N}_{l}=\mathrm{N}_{2}=35\right)$. 
When flamingo partners were on the nest, we compared the time spent standing and

178 sitting on the nest between female and male flamingos. When on the nest, females spent on average $5.5 \%$ of their time standing and $94.5 \%$ sitting whereas males spent on average $3.9 \%$ of their time standing and $96.1 \%$ sitting (see Tab. 2 for medians and IQR). Mann-Whitney tests revealed that males spent significantly more time than females sitting on the nest $(U=375, P=$ $0.005, \mathrm{~N}_{l}=\mathrm{N}_{2}=35$ ), whereas no significant differences were found in the time spent standing on the nest $(U=602, P=0.905)$.

Female and male activity near the nest and on the nest

When flamingo partners were near the nest, the behavioural categories observed were agonistic behaviour, self-directed comfort behaviour, sleeping and other activities not directly associated with parental care ("other") (Tab. 2). Mann-Whitney tests revealed that when near the nest males spent significantly more time than females performing the behavioural categories mentioned above: agonistic behaviour $\left(U=300.5, P=0.0003, \mathrm{~N}_{l}=\mathrm{N}_{2}=35\right)$, self-directed comfort behaviour $\left(U=319.5, P=0.0006, \mathrm{~N}_{l}=\mathrm{N}_{2}=35\right)$, sleeping $\left(U=229, P<0.0001, \mathrm{~N}_{l}=\mathrm{N}_{2}=35\right)$ and “other" $\left(U=198.5, P<0.0001, \mathrm{~N}_{l}=\mathrm{N}_{2}=35\right)$. observed were agonistic behaviour, egg-care related behaviour (egg-care) and self-directed comfort behaviour (Tab. 2). Mann-Whitney tests revealed that males spent significantly less time than females in self-directed comfort behaviour $\left(U=416.5, P=0.021, \mathrm{~N}_{l}=\mathrm{N}_{2}=35\right)$, whereas no significant differences were found for agonistic behaviour $\left(U=600, P=0.889, \mathrm{~N}_{I}=\mathrm{N}_{2}=35\right)$ and egg-care $\left(U=515, P=0.254, \mathrm{~N}_{l}=\mathrm{N}_{2}=35\right)$. 
200 (preening) and sleeping (Tab. 2). Mann-Whitney tests revealed that males spent significantly

201 more time than females in agonistic behaviour $\left(U=358.5, P=0.003, \mathrm{~N}_{l}=\mathrm{N}_{2}=35\right)$, whereas no

202 significant differences were found for attentive behaviour $(U=460, P=0.073)$, nest-building $(U$

$\left.203=474, P=0.105, \mathrm{~N}_{l}=\mathrm{N}_{2}=35\right)$, self-directed comfort behaviour $\left(U=571, P=0.631, \mathrm{~N}_{l}=\mathrm{N}_{2}=35\right)$

204 and sleeping $\left(U=551.5, P=0.477, \mathrm{~N}_{l}=\mathrm{N}_{2}=35\right)$.

205 DISCUSSION \& CONCLUSION

206 This study aimed at investigating the parental care behaviour of female and male greater

207 flamingos, focusing on the time spent by each partner in different positions in relation to the nest 208 and performing different behaviours. First, our results suggested that male flamingos spent more

209 time on the nest and near it than females, whereas females stayed away from the nest $(>150 \mathrm{~cm})$

210 longer than males. The second main finding of this study was that male flamingos, when near the

211 nest and when sitting on the nest, performed more aggressive behaviour toward conspecifics

212 approaching the nest than females, protecting more intensively the egg and the nesting site.

213 Research on flamingo breeding behaviour is needed to improve the knowledge on these

214 species, to find strategies that can improve their husbandry in captivity and safeguard their

215 welfare (Ogilvie \& Ogilvie, 1986; Hosey et al., 2013). The aim of this study was to investigate

216 the parental behaviour of a captive colony of greater flamingo to improve the knowledge on this

217 species in zoological gardens. Firstly, greater flamingos of this study were found to perform

218 species-specific behavioural repertoire (Brown \& King, 2005). In particular, both flamingo

219 partners were involved in parental care and displayed all the activities reported in the wild during

220 incubation, such as moving and rotating the egg, nest-building, self-preening and stretching, nest

221 protection and resting (Studer-Thiersch, 1975; Pickering et al., 1992; Brown \& King, 2005;

222 Beletsky, 2006; Elphick, 2014). 
224 female and male greater flamingos. Firstly, male flamingos of a breeding pair spent significantly

225

227

228

229

230

231

232

233

234

235

236

237 more time on the nest and near it than females. These finding are in agreement with previous studies reporting a greater effort of male greater flamingos in incubation in wild settings (Rendòn-Martos et al., 2000; Rendòn, Garrido, Rèndon-Martos, Ramirez \& Amat, 2014). On the contrary, females remained away from the nest, without caring about the egg and the nest, longer than males. On the basis of previous studies, male flamingos take care of the egg but do not feed their partner during the incubation process. Moreover, at least in the early stages, the parental investment is greater for females than males, due to the costs of egg-laying (Cezilly, 1993; Johnson \& Cezilly, 2009). Although we did not collect data on flamingo behaviour away from the nest, it might be possible that since females remained less time on the nest and in proximity of the nest they spent more time looking for food, to recover from the egg-laying effort and replenish their reserves. Similar findings have been found in studies on breeding biology and parental care in shorebirds (Lenington, 1984; Reynolds \& Szèkely, 1997). Further studies focusing on the behaviours performed by each partner away from the nest are needed to add data supporting this hypothesis.

When flamingos were on the nest, they could either be standing or sitting to incubate the egg. Our findings suggest that male flamingos spend more time sitting on the nest, thus incubating the egg than females, although no differences between sexes for standing on the nest were reported. Together with previous results on nest attendance (being near the nest or on the nest), these findings suggest a greater involvement of male flamingos in the incubation process as previously reported in wild settings (Rendòn-Martos et al., 2000; Rendòn et al., 2014). 
When flamingos were near the nest, males were significantly more aggressive at

246

247

defending the nest from other individuals than females and performed more self-comfort

behaviour, sleeping and other behavioural categories. It might be possible that, since males were more involved in nest defence, they remained near the nest for a longer time when their partner was on the nest, instead of going away similarly to females.

When flamingos were standing on the nest the most important behaviour was caring for the egg, moving or rotating it to improve the incubation effort. According to our results, females and males spent the same amount of time in the egg care, confirming previous findings on parental care in greater flamingos (Studer-Tiersch, 1975; Elphick, 2014) and Caribbean flamingos (Brown et al., 1983) in zoological gardens.

Finally, even when flamingos were sitting on the nest, incubating the egg, males spent significantly more time than females performing agonistic behaviour. Aggressive behaviour has been previously found to increase during the breeding season in flamingos (Farrell et al., 2000) and might be due to competition over mates and over nest sites and food resources, as well as for nest/chick protection (Johnson \& Cèzilly, 2009; Hinton et al., 2013). According to our results, both female and male flamingos displayed agonistic behaviour. However, male flamingos were more aggressive than females when they were either near the nest and on the nest, incubating the egg. The study greater flamingos performed aggressive behaviours such as pecking at other birds or threat displays such as neck hooking and swaying (Studer-Thiersch, 2000; Johnson \& Cèzilly, 2009; Schmitz \& Baldassarre, 1992) mainly to keep other birds away from their nest. Thus, our findings suggest that male flamingos are also largely involved in nest/chick protection. In the current study, we focused on the parental care behaviour of the partners after the egg was laid and the nest was almost completed. The lack of differences in nest building between 
268 sexes reported in the current study seems to confirm that during incubation, after the egg is laid,

269 nest building duties are equally shared by both partners, as reported in previous research on zoo

270 greater flamingos (Studer-Tiersch, 1975).

271 In wild greater flamingos, the hatchling success is about $30 \%$ due to a high frequency of

272 predation and other threats, almost absent in captivity (Brown \& King, 2005). In the current

273 study, $77 \%$ of the breeding pairs had a chick. Moreover, although wild flamingos lay one egg per

274 breeding season, if it fails to hatch or the chick dies in the early stages, another egg might be laid

275 (Pickering, 1992). In our study, only two pairs (7.4\%) had to lay more than once before obtaining

276 a successful hatch. Taken together, these findings suggest that more than three quarters of the

277 eggs hatched and relatively few flamingo pairs had to lay more eggs before having a chick.

278 Parental care of the study colony seems therefore to be linked to a good pair productivity in

279 terms of number of eggs laid, as only one deposition is generally sufficient to have a chick.

280 Future studies should be done to better investigate the relationship between greater flamingo

281 parental care behaviour and their reproductive success.

Studies on greater flamingo social biology and pair bonding are relevant to enhance our

understanding of the ethology of this species (Rose et al., 2014). Our data on the parental care of

female and male greater flamingos can be added to the previous literature on this species. This

kind of research is important not only to expand the knowledge on bird species such as

286

flamingos, but also to improve the situation of captive animals and have a greater scientific

287

288

289

290 understanding of issues important to modern zoos and ex-situ conservation. Indeed, speciestypical behaviours of the animals are strongly informative of their feelings, preferences and needs and might therefore provide useful information to detect and address welfare issues (e.g.: abnormal behaviour) and to enhance their daily lives (Mench, 1998). 


\section{Acknowledgement}

292 We would like to thank Dr. Cesare Avesani Zaborra for allowing this study to take place in Parco

293 Natura Viva. Furthermore, special thanks should be given to Giuseppe Alampi, Parco Natura

294 Viva flamingo zoo-keepers, for his hard work and for their precious help with the flamingo

295 census and monitoring. Finally, we thank Paul Rose, Sunny Nelson and Jean-Loup Rault for

296 their careful review of our manuscript and the useful comments and suggestions.

\section{REFERENCES}

298 Altmann J. 1974. Observational study of behaviour: sampling methods. Behaviour 49:227-267.

299 DOI: $10.1163 / 156853974 X 00534$.

300

301

302

Bagemihl B. 1999. Biological exuberance: Animal Homosexuality and Natural Diversity.

NewYork, NY: St. Martin’s Press.

303

304

Beletsky L. 2006. Birds of the World. Baltimore, MD: The John Hopkins University Press.

305

306

Brown C, King C. 2005. Flamingo husbandry guidelines; a joint effort of the AZA and EAZA in

cooperation with WWT. Dallas, TX: Dallas Zoo. Available at

308

http://www.flamingoresources.org/husbandry.html

309

310

Brown SG, Shannon P, Farnell G. 1983. Renesting and brooding in the Caribbean flamingo. Zoo

311

Biology 2:137-141. DOI: 10.1002/zoo.1430020207

312 
313 Cézilly F. 1993. Nest desertion in the great flamingo, Phoenicopterus ruber roseus. Animal

314 Behavior 45:1038-1040. DOI: http://dx.doi.org/10.1006/anbe.1993.1125

315

316 Elphick J. 2014. The World of Birds. Buffalo, NY, London, United Kingdom, and Ontario, 317 Toronto: Firefly Books.

318

319 Farrell MA, Barry E, Marples N. 2000. Breeding behavior in a flock of Chilean flamingos 320 (Phoenicopterus chilensis) at Dublin Zoo. Zoo Biology 19:227-237. DOI: 10.1002/10982361(2000)19:4<227::AID-ZOO1>3.0.CO;2-H.

Hauber ME 2014. The Book of Eggs: A Life-Size Guide to the Eggs of Six Hundred of the

World's Bird Species, Chicago: University of Chicago Press.

325

326 Hinton MG, Bendelow A, Lantz S, Wey TW, Schoen L, Brockett R, Karubian J. 2013. Patterns 327 of aggression among captive American flamingos (Phoenicopterus ruber). Zoo Biology 9999:1328 9. DOI: 10.1002/zoo.21078.

Hosey, G., Melfi, V., \& Pankhurst, S. (2013). Zoo Animals: Behaviour, Management, and 331 Welfare-Second Edition. Oxford, UK: Oxford University Press.

332 333 del Hoyo J, Elliott A \& Sargatal J. 1992. Handbook of the Birds of the World. Volume 1: Ostrich to Ducks. Barcelona: Lynx Edicions. 
336 Johnson AR, Cézilly F. 2009. The Greater Flamingo. London, UK: T \& AD Poyser.

337

338 King CE. 2000. Captive flamingo populations and opportunities for research in zoos.

339 Waterbirds: The International Journal of Waterbird Biology 23:142-149. DOI:

$340 \quad 10.2307 / 1522159$.

341

342 Lenington S. 1984. The evolution of polyandry in shorebirds. In: Burger J. and Olla BL, eds.

343 Shorebirds: Breeding Behavior And Populations. New York: Plenum Press, 149-167.

344

345

Melfi V. 2009. There are big gaps in our knowledge, and thus approach, to zoo animal welfare: a case for evidence-based zoo animal management. Zoo Biology 28:574-588. DOI:

347

10.1002/zoo.20288.

Mench JA. 1998. Why it is important to understand animal behavior. ILAR JOURNAL 39:20-26

350

351

Ogilvie M, Ogilvie C. 1986. Flamingos. Gloucester: Alan Sutton.

352

353

Pickering, S. 1992. The comparative breeding biology of flamingos, Phoenicopteridae, at the 354

Wildfowl and Wetlands Trust Centre, Slimbridge. International Zoo Yearbook 31:139-46. DOI:

355 http://dx.doi.org/10.1111/j.1748-1090.1991.tb02377.x

356

357 Pickering S, Creighton E, Stevens-Wood B. 1992. Flock size and breeding success in flamingos.

358 Zoo Biology 11:229-234. DOI: 10.1002/zoo.1430110402. 
Rendón MA, Garrido A, Rendón-Martos M, Ramírez JM, Amat JA. 2014. Assessing sex-related chick provisioning in greater flamingo Phoenicopterus roseus parents using capture-recapture models. Journal of Animal Ecology 83:479-490. doi: 10.1111/1365-2656. DOI: 10.1111/13652656.12138 .

Rendón-Martos M, Vargas JM, Rendón MA, Garrido A, Ramírez JM. 2000. Nocturnal movements of breeding greater flamingos in southern Spain. Waterbirds 23 (Special Publication 1):9-19.

Reynolds JD, Szèkely T. 1997. The evolution of parental care in shorebirds: life histories, ecology and sexual selection. Behavioral Ecology, 8:126-134. DOI: 10.1093/beheco/8.2.126.

Rose PE, Croft DP, Lee R. 2014. A review of captive flamingo (Phoenicopteridae) welfare: a synthesis of current knowledge and future directions. International Zoo Yearbook 48:139-155. DOI: $10.1111 /$ izy.12051

375

Rose PE \& Croft, DP. 2015. Evidence of directed interactions between individuals in captive flamingo flocks. Wildfowl 65:123-138.

379 Sandri C, Sammarini, C, Regaiolli B, Spiezio C \& Piccirillo, A. Reproduction and Monogamy in 380 a Captive Flock of Greater Flamingos (Phoenicopterus roseus). Journal of Applied Animal 381 Welfare Science, submitted. 
383 Schmitz, RA \& Baldassarre, GA. 1992. Contest asymmetry and multiple bird conflicts during 384 foraging among nonbreeding American flamingos in Yucatan, Mexico. The Condor 94:254-259

385

386 Studer-Tiersch A. 1975. Basle Zoo. In: Johnson A. and Cezilly F. eds. The Greater Flamingo.

387 Berkamsted, UK: T \& A D Poyser, 121-130.

388

389 Studer-Thiersch A. 2000. What 19 years of observation on captive greater flamingos suggests

390 about adaptations to breeding under irregular conditions. Waterbirds: The International Journal 391 of Waterbird Biology 23:150-159.

392

393

394

395

396

397

398 


\section{Table 1 (on next page)}

Behavioural ethogram of the study

For each behavioural category collected in the study the table reports the name and definition. 


\section{Behavioural categories}

Agonistic

During nesting, a flamingo extends the neck and peck at another bird or performs threatening behaviour displays, particularly neck hooking and swaying (Studer-Thiersch, 2000; Johnson \& Cèzilly, 2009) and feather spreading (Schmitz \& Baldassarre, 1992). Agonistic behaviour can be performed when the flamingo is near the nest and on the nest (both standing and sitting).

Incubation

A flamingo is incubating the egg, keeping it warm with the body heat. This behaviour is performed when the flamingo is sitting on the nest.

Comfort

A flamingo is self-preening (trimming or dressing the own feathers with the beak), stretching behaviour (drawing out/extending legs or wings) or scratching the neck with one leg (Studer-Thiersch, 1975; Brown \& King, 2005). Comfort behaviour can be performed when the flamingo is near the nest and on the nest (both standing and sitting).

Egg care

A flamingo is looking at the egg or rolling and moving the egg carefully (Studer-Thiersch, 1975; Brown \& King, 2005), to assure even heating or cooling and prevent embryonic malformation (Hauber, 2014). Egg care can be performed when the flamingo is standing on the nest.

Nest-building

A flamingo looks for mud, soil and sand and picks them up to renovate or repair the nest (StuderThiersch, 1975; Brown \& King, 2005). Nest-building can be performed when the flamingo is sitting on the nest.

Sleeping

A flamingo rests with the head under the wing. Sleeping can be performed when the flamingo is near the nest or sitting on the nest.

Other

A flamingo performs a behaviour that is not directly associated with parental care and is not listed above. This behavioural category can be performed when the flamingo is near the nest. 


\section{Table 2 (on next page)}

Behavioural categories performed by flamingos near the nest and on the nest (standing and sitting)

The table reports the median (IQR) duration in seconds of each behavioural category

performed by females $(F)$ and males $(M)$ when they were near the nest, standing on the nest or sitting on the nest, incubating the egg. The last row reports the median (IQR) duration in seconds of time spent by female and male flamingos in different position. 


\begin{tabular}{|c|c|c|c|c|c|c|}
\hline & \multicolumn{2}{|l|}{ Near the nest } & \multicolumn{2}{|c|}{ On the nest (standing) } & \multicolumn{2}{|l|}{ On the nest (sitting) } \\
\hline & $\mathbf{F}$ & $\mathbf{M}$ & $\mathbf{F}$ & $\mathbf{M}$ & $\mathbf{F}$ & $\mathbf{M}$ \\
\hline Agonistic behaviour & $40(3-105.5)$ & $187(40-326)$ & $11(0-21)$ & $8(0-19)$ & $545(375-884)$ & $921(637-1105.5)$ \\
\hline Comfort behaviour & $231(27-480)$ & $524(210-945)$ & $14(0-71.5)$ & $0(0-24)$ & $64(0-165.5)$ & $59(4-230.5)$ \\
\hline Sleeping & $55(0-434)$ & $934(358-1378)$ & - & - & $67(0-634)$ & $319(0-714.5)$ \\
\hline Egg care & - & - & $148(72-239.5)$ & $172(99-320)$ & - & - \\
\hline Incubation & - & - & - & - & $1650(1081-1895)$ & $1995(1181-2578.5)$ \\
\hline Nest-building & - & - & - & - & $2336(1523-2956)$ & 2791 (2036-3469) \\
\hline Other & $255(71-502)$ & $1093(432-1836.5)$ & - & - & - & - \\
\hline Position & $763(287-1405.5)$ & $2862(1654-4365.5)$ & $168(114-380)$ & $228(99-385)$ & $5464(4010-6067.5)$ & $6000(5238-7248)$ \\
\hline
\end{tabular}




\section{Figure 1}

Box and whisker plot of the time spent by flamingo partners near the nest

The horizontal lines within the box indicate the medians, boundaries of the box indicate the $25^{\text {th }}$ and $75^{\text {th }}$ percentile and the whiskers indicate the minimum and maximum values of the data samples. Outliers are drawn as points.

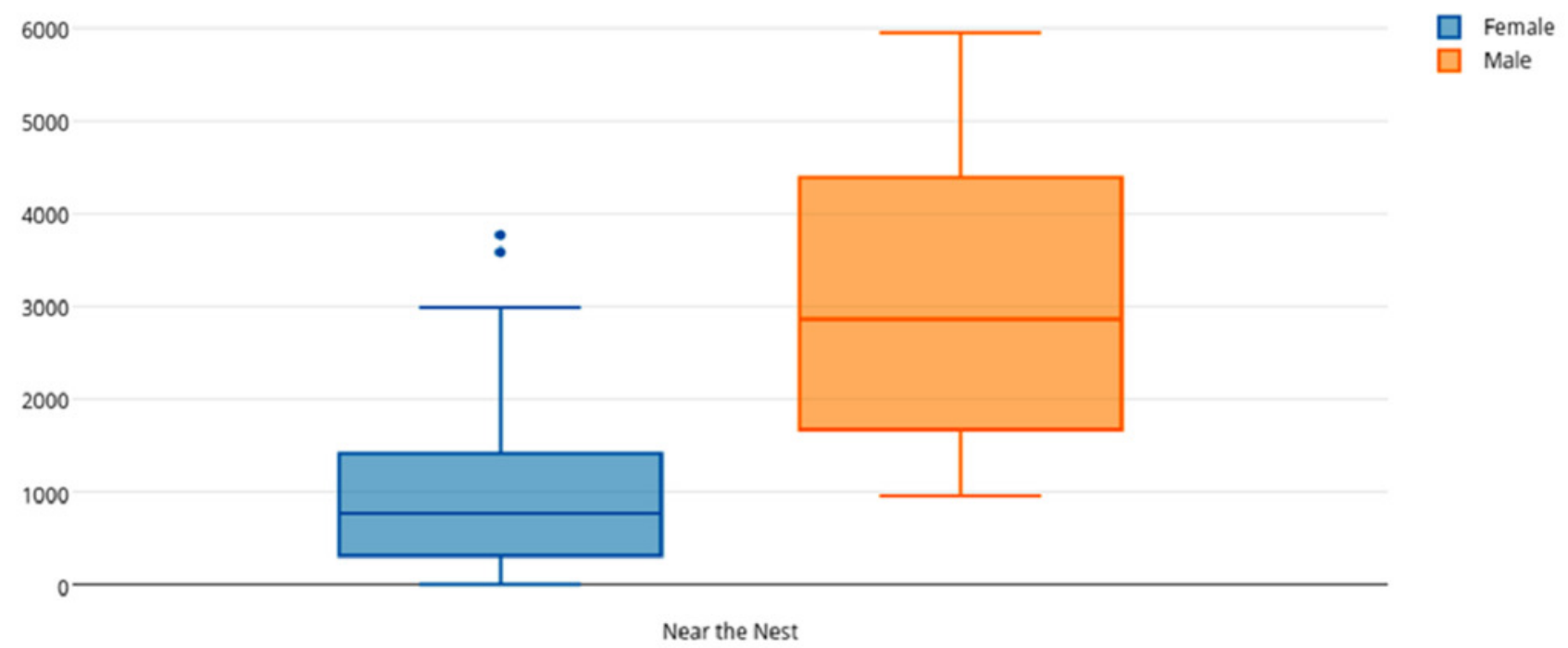


Figure 2

Box and whisker plot of the time spent by flamingo partners on the nest

The horizontal lines within the box indicate the medians, boundaries of the box indicate the $25^{\text {th }}$ and $75^{\text {th }}$ percentile and the whiskers indicate the minimum and maximum values of the data samples. Outliers are drawn as points.

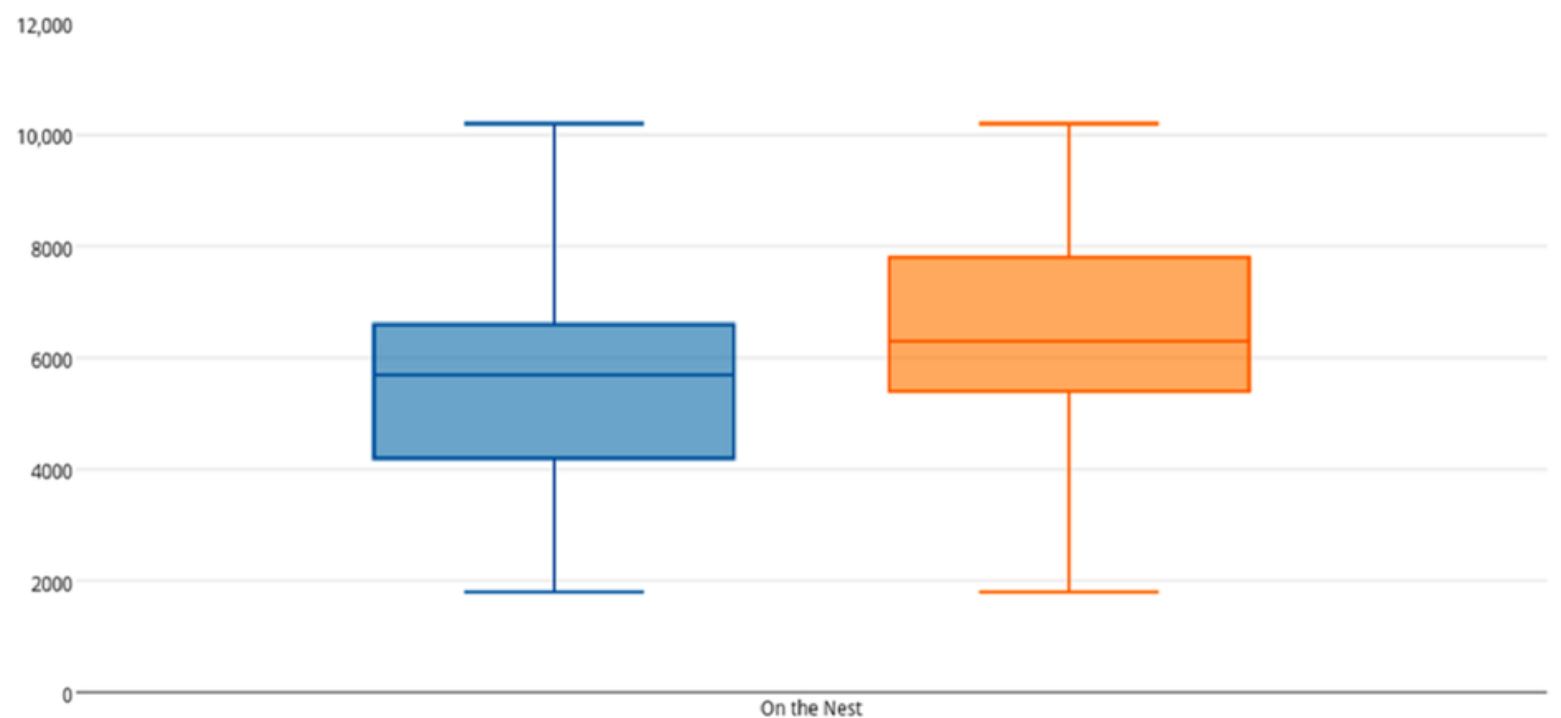


Figure 3

Box and whisker plot of the time spent by flamingo partners away from the nest (No Nest)

The horizontal lines within the box indicate the medians, boundaries of the box indicate the $25^{\text {th }}$ and $75^{\text {th }}$ percentile and the whiskers indicate the minimum and maximum values of the data samples. Outliers are drawn as points.

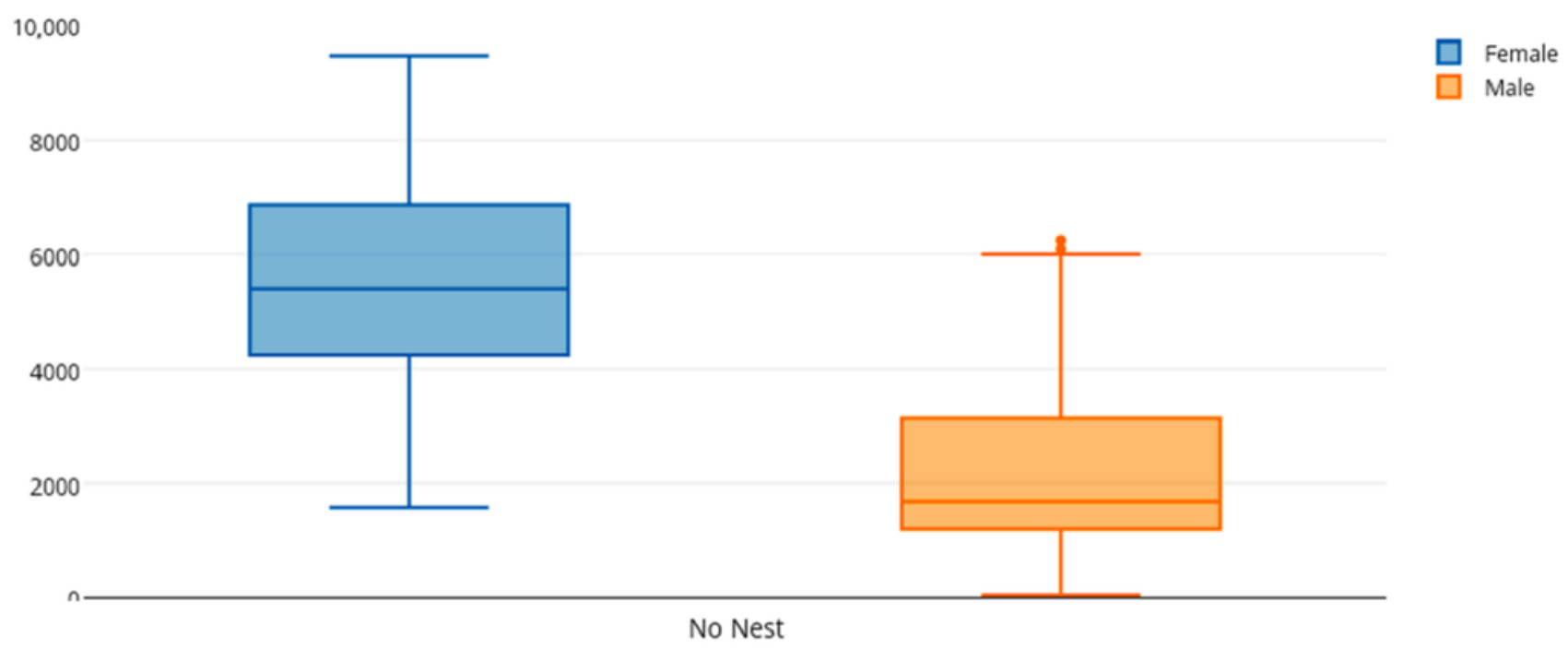

